\title{
Properties of Fiberboards Bonded by Decomposed Polyurethane Foams and Isocyanate
}

\author{
Tongtong Cui ${ }^{1}$, Yan $\mathrm{Li}^{1}$, Yanfang Pang ${ }^{1}$, Xiaosheng Liu ${ }^{1}$, Huidong $\mathrm{Su}^{1}$, An Mao ${ }^{1,2, *}, \mathrm{Qi} \mathrm{Li}^{1}{ }^{\text {, }}$, \\ Yifu Yuan, ${ }^{1, *}$ \\ ${ }^{1}$ Key Laboratory of State Forestry Administration for Silviculture of the Lower Yellow River, College of Forestry, Shandong Agricultural \\ University, Taian, China \\ ${ }^{2}$ Beijing Key Laboratory of Wood Science and Engineering, Beijing Forestry University, Beijing, China
}

\section{Email address:}

dannymaoan@126.com (An Mao), wonderfulliqi2364@126.com (Qi Li), yuanyf16@163.com (Yifu Yuan)

${ }^{*}$ Corresponding author

\section{To cite this article:}

Tongtong Cui, Yan Li, Yanfang Pang, Xiaosheng Liu, Huidong Su, An Mao, Qi Li, Yifu Yuan. Properties of Fiberboards Bonded by Decomposed Polyurethane Foams and Isocyanate. American Journal of Agriculture and Forestry. Vol. 7, No. 6, 2019, pp. 315-320. doi: 10.11648/j.ajaf.20190706.21

Received: November 7, 2019; Accepted: November 22, 2019; Published: December 4, 2019

\begin{abstract}
The objective of this study was to investigate the feasibility of using recycled polyols as part of the binder system for wood panels (fiberboard). PU foam wastes from disposed insulation materials were collected, processed, and chemically decomposed by agent to obtain the recycled polyol. The recycled polyol was mixed with a commercial polyol at various weight ratios $(0: 100,20: 80,40: 60,60: 40,80: 20$, and 100:0) to prepare a series of polyol mixtures. Then, the obtained polyol mixtures were then sprayed successively with pMDI resin at various pMDI/polyol mixtures weight ratios (100: 0, 75: 25, and 50: 50) onto wood fibers to prepare fiberboards. The effects of recycled polyol/commercial polyol weight ratio and pMDI/polyol mixture weight ratio on physical and mechanical properties of bonded fiberboards were evaluated. The results showed that the addition of recycled polyol into a commercial polyol helped improve the IB, MOR, and MOE strength of fiberboard, but their effects on water resistance of fiberboard were minor. With the increase of percentage of polyol mixture in $\mathrm{pMDI} /$ polyol mixture, the properties of fiberboard presented a trend of decreasing. When the recycled polyol/commercial polyol weight ratio was 40: 60 and the pMDI/polyol mixture was 25: 75, the bonded fiberboard had the overall best physical and mechanical performances. The application of decomposed PU foams in fiberboard production provided a new possible way of recycling polymer wastes.
\end{abstract}

Keywords: Polyurethane Foam, Recycle, Decomposition, Bonding, Isocyanate

\section{Introduction}

Polyurethanes (PU) are synthesized polymers containing urethane groups (-NH-COO-) or isocyanate group (-NCO) in their main molecule chains. They are usually made by reacting diisocynates and polyols with the addition of some catalysts and agents. As widely used high molecular weight materials, the amount of disposed PU foams is also huge every year. PU foams are excellent insulation materials because of their foaming structures which can provide good heat insulation property. However, when the insulation materials reach the end of their service lives, they are usually disposed and could not be recycled or reused in an easy way.
Because they have strong chemical bonds that could not degraded under natural conditions. The traditional recycling methods of PU foams are land filling and burning. However, the land filling of PU foams takes up too much space and still has negative impact on the environment. Moreover, burning of PU foams could recycle heat energy, but also would generate some harmful gases. Therefore, new recyclingmethods of PU foam are needed to solve the current environment problem [1].

Some potential recycling methods are reported in recent years including physical, chemical, and energy methods [2]. Each method contains different processes. PU foams can be chemically decomposed into feedstock chemicals in the 
presence of agent under high temperature. The agents can be water, alcohols, inorganic or organic acids, amines, and alkalines [3-5]. The polyols from the decomposed PU foams can be recycled and reused for formulating new PU products. For example, the recycled polyols can be used for bonding wood panel products $[6,7]$.

The wood panel industry is developing quickly in China with the annual production reaching around three thousand million cubic meters. The primary adhesive used for bonding wood panels is urea-formaldehyde (UF) resin [8-10]. However, UF-bonded panels have problems of formaldehyde emission and low water resistance [11]. Therefore, panels with no formaldehyde emission and higher water resistance are needed [12].

The objective of this study was to investigate the feasibility of using recycled polyolsas part of the binder system for wood panels (fiberboard). The recycled polyols were obtained by decomposing PU foams under action of dipropylene glycol (DPG) and mixed with a commercial polyether polyol at various weight ratios to obtain a series of polyol mixtures. Fiberboards were made by applying polyol mixtures and pMDI resin successively on the fibers at different weight ratios. The effects of recycled polyol/commercial polyol weight ratio and $\mathrm{pMDI} /$ polyol mixture weight ratio, and adhesive application rate on physical and mechanical properties of fiberboards were studied.

\section{Materials and Methods}

\subsection{Materials Preparation}

PU foams were obtained from a local recycling store. They were picked from insulation layer of disposedrefrigerators. The original color of the foams was light yellow. The foams were washed with water and acetone, and then were dried naturally. After the foams were air-dried, they were grounded into powders of 60 mesh with a laboratory grounding machine (Model WQM, Jingcheng Chemical Machinery Co. Ltd, Jiangyin). The obtained PU powders were then dried in an oven at $100^{\circ} \mathrm{C}$ until all water was removed.

DPG and potassium hydroxide used as decomposing agent and catalyst, respectively, were obtained from Sinopharm Group Co. Ltd, Beijing. DPG is a low viscosity, transparent liquid with a water content of up to $0.5 \%$ and a hydroxyl number of about $836 \mathrm{mg} \mathrm{KOH} / \mathrm{g}$. Acetone was also obtained from Sinopharm Group Co. Ltd, Beijing. PMDI (WANNATE PM-200) with the NCO content of $30.5 \%-32 \%$ was obtained from Wanhua Chemical Group Co., Ltd, Shandong, China. It was a dark yellow liquid with a viscosity of $150-250 \mathrm{mPa} \cdot \mathrm{s}$ at $25^{\circ} \mathrm{C}$. Commercial polyether polyol (WANOLC2056) with thehydroxyl group value of 54-57 $\mathrm{mg} \mathrm{KOH} / \mathrm{gwas}$ also obtained from Wanhua Chemical Group Co., Ltd, Shandong, China. It was a light yellow liquid with a viscosity of $270-370 \mathrm{mPa} \cdot \mathrm{s}$ at $25^{\circ} \mathrm{C}$. Mixed pine wood fibers with an original moisture content of $12 \%-15 \%$ were obtained from Toroyal Wood Products Corp., Shandong, China.

\subsection{Decomposition of PU Foams}

The PU foam decomposition reaction was carried out under the atmosphere pressure in a four-neck round-bottom flask equipped with astirrer and arefluxing condenser. A two-blade Polyethylene Terephthalate (PETE) stirrer was used to stir the reaction mixture in the flask. The calculated amount of DPG was placed in the flask and heated by a heating mantle to $180^{\circ} \mathrm{C}$. Then, the catalyst (potassium hydroxide) and PU foam powders were charged in the flask successively. After the charging was completed, the reaction mixture was maintained at $180^{\circ} \mathrm{C}$ for $2.5 \mathrm{~h}$. The obtained reaction mixture was transferred to a separating funnel and split into two phases after cooling down to room temperature. The upper phase was separated and restored as the recycled polyol.

Table 1. Decomposition parameters of PU foam powders.

\begin{tabular}{ll}
\hline Reaction pressure & Atmospheric pressure \\
\hline Reaction temperature $\left({ }^{\circ} \mathrm{C}\right)$ & 180 \\
Reactiontime $(\mathrm{h})$ & 2.5 \\
Decomposition agent & $\mathrm{DPG}$ \\
PU/agent weight ratio & $3: 1$ \\
Catalyst & Potassium hydroxide \\
Catalyst concentration $(\%)$ & 1.0 \\
\hline
\end{tabular}

\subsection{Property Measurements of Recycled Polyol}

Hydroxyl number was measured in accordance with the AOCS Cd 13-60 standard. Pyridine, acetic anhydride, pyridine-acetic anhydride, normal butyl alcohol, ethanolic potassium hydroxide, and the phenolphthalein indicator solution were obtained from Sinopharm Group Co. Ltd, Beijing. Three replicates were used and averaged for each measurement. The hydroxyl number was calculated as follows:

Hydroxyl number $=\{[\mathrm{B}+(\mathrm{W} \times \mathrm{A} / \mathrm{C})-\mathrm{S}] \times \mathrm{N} \times 56.1\} / \mathrm{W}(1)$

Where:

Ais the $\mathrm{KOH}$ solution required for the acidity titration $(\mathrm{mL})$,

$\mathrm{B}$ is the $\mathrm{KOH}$ solution required for the reagent blank $(\mathrm{mL})$, $\mathrm{C}$ is the weight of sample used for the acidity titration $(\mathrm{g})$,

$\mathrm{S}$ is the $\mathrm{KOH}$ solution required for titration of the acetylated sample $(\mathrm{mL})$,

$\mathrm{W}$ is the weight of sample used for acetylation ( $\mathrm{g})$,

$\mathrm{N}$ is the normalityof the $\mathrm{KOH}$ solution $(\mathrm{N})$.

Viscosity was measured with a Rotational Brookfield Viscometer (Brookfield Engineering Laboratories. Inc., Middleboro, MA). The measurements were performed at $25^{\circ} \mathrm{C}$.

Amine number was measured in accordance with the procedures described in ASTM D2073. Acetic acid, acetic anhydride, chloroform, hydrochloric acid, isopropanol, perchloric acid, and salicylaldehyde were obtained from Sinopharm Group Co. Ltd, Beijing. Three replicates were used and averaged for each measurement. The total amine number was calculated as follows: 


$$
\text { Total amine number }=(\mathrm{V} \times \mathrm{N} \times 56.1) / \mathrm{S}
$$

Where:

$\mathrm{V}$ is the amount of hydrochloric acid required for titration of the sample $(\mathrm{mL})$,

$\mathrm{N}$ is the normality of the hydrochloric acid solution $(\mathrm{N})$,

$\mathrm{S}$ is the sample weight used $(\mathrm{g})$.

$\mathrm{PH}$ value was determined with an Accumet basic AB15 pH meter (Thermo Fisher Scientific, Waltham, MA) at $25^{\circ} \mathrm{C}$.

\subsection{Fiberboard Manufacturing}

Mixed pine wood fibers were first dried to the moisture content of $6.0 \%$ and put into a laboratory rotating blender. Then, the polyol mixtures and pMDI resin was calculated and pumped via a tube, and sprayed on the rotating fiberssuccessively. The blending time was about $10 \mathrm{~min}$. The blended fibers were weight and hand-laid on a stainless steel caul plate in a formatting box with a dimension of $500 \mathrm{~mm} \times$ $500 \mathrm{~mm}$. A uniform wood fiber mat was obtained. After removing the box, the mat was compressed and another stainless steel caul plate was put on the compressed mat. Then, the mat was transferred to a laboratory hot press. The hot press time and temperature were $4.5 \mathrm{~min}$ and $180^{\circ} \mathrm{C}$, respectively. After hot pressing, the fiberboards were conditioned at $20^{\circ} \mathrm{C}$ and 60 percent relative humidity for 1 week. The experimental design and fiberboard manufacturing parameters were shown in Table 2.

Table 2. Fiberboard manufacturing parameters.

\begin{tabular}{ll}
\hline Recycled/commercial polyol & $0: 100,20: 80,40: 60,60: 40,80:$ \\
PMDI/polyol mixture & $20,100: 0$ \\
Adhesive application rate $(\%)$ & $100: 0,75: 25,50: 50$ \\
Moisture content of fibers $(\%)$ & 5 \\
Hot press temperature $\left({ }^{\circ} \mathrm{C}\right)$ & 6 \\
Hot press time (min) & 180 \\
Hot press pressure $(\mathrm{MPa})$ & 4.5 \\
\hline
\end{tabular}

\subsection{Physical and Mechanical Testing of Particleboards}

Density of each test sample was obtained by dividing weight by volume. Internal bond (IB), modulus of rupture (MOR) and modulus of elasticity (MOE) were tested on a CMT 4104 universal testing machine (MTS System Corporation, MN). Water-soak thickness swell (TS) and water absorption (WA) values were measured in a $20^{\circ} \mathrm{C}$ water bath according to Chinese National Standard GB/T 17657-2013. Six IB samples, three bending samples and two water-soak samples were tested from each board.

\section{Results and Discussion}

\subsection{The General Properties of Recycled Polyol}

The general properties of the recycled polyols were shown in Table 3 . The hydroxyl number of the recycled polyol was $118 \mathrm{mg} \mathrm{KOH} / \mathrm{g}$, the viscosity was $519 \mathrm{mg}$ $\mathrm{KOH} / \mathrm{g}$, the amine number was $2.08 \mathrm{mg} \mathrm{KOH} / \mathrm{g}$, and the
$\mathrm{pH}$ value was 7.6, respectively. The hydroxyl number of the polyol could be largely influenced by the choice of the decomposition agent [4]. In this study, the reaction mixtures obtained from the decomposition reaction conditions were subjected to the separation to obtain recycled polyols with uniform phase. In this case, the influence of the decomposition agent (DPG) on the hydroxyl number of the recycledpolyols was minor.

As the reaction progressed, the PU chains were attacked by the DPG, and got broken, and the molecular weight of the polymer thus decreased resulting in a lower viscosity value. The viscosity of the recycled polyols is strongly depended on the reaction temperature. In this study, the reaction temperature of $180^{\circ} \mathrm{C}$ is appropriate for the decomposition of PU powders. Other than the reaction temperature, the viscosity of the recycledpolyols could also be influenced by the residual DPG and other by-products derived from the aromatic segments of the isocyanate.

Amine number indicates the concentration of the amine compounds in the polyol. The amine compounds usually come from the hydrolysis of the urethane bonds [4]. The hydrolysis is unavoidable in decomposition reaction. Although PU foam powders were oven-dried to remove all the water, the DPG still contains up to $0.5 \%$ of water. Another possible source of the amine compounds might be the decomposition of the urea bonds in the PU foams. The urea bonds were formed by the reaction between water and isocyanate during PU formulation reaction. Although the amount of the urea bonds was minor, they could still affect the properties of the recovered polyols. If the recovered polyols were reused in the preparation of new PU products, the amine compounds might negatively affect the PU foaming reaction. For example, they could accelerate the reaction and form undesirable urea bonds. In this study, once the recycled polyol was mixed with pMDI resin, fast reaction could happen in a short time because of the presence of amine compounds in the recycled polyol. Therefore, the recycled polyol and pMDI resin had to be applied separately onto the fibers.

The $\mathrm{pH}$ value of the recycled polyols was in the normal range of 7.5-8.0. The minor basic condition was probably from the catalyst used in decomposition reaction.

\subsection{Comparison of Recycled Polyol with Commercial One}

The application of the recycled polyols was usually in preparation of the PU foams. However, the recycled polyols could only partially $(5-20 \%)$ replace the commercial ones toobtain PU foams with satisfied properties. In this study, the general properties of recycled polyol were compared with a commercially used polyether polyol (WANOLC2056) (Table 3). After comparison, the properties of obtained recycledpolyols were similar to those of the commercial one which have been used in the preparation of PU coatings, elastomers, adhesives, and sealants. Therefore, it is proposed that the recycledpolyols obtained in this study may be suitable for the preparation of adhesives for wood panels such as fiberboard. 
Table 3. General properties of recycled and commercial polyols.

\begin{tabular}{lll}
\hline Properties & Recycled polyol & Commercial polyol \\
\hline Appearance & Light grey & Light yellow \\
Hydroxyl number $(\mathrm{mg} \mathrm{KOH} / \mathrm{g})$ & 68 & 57 \\
Viscosity $(\mathrm{mPa} \cdot \mathrm{s})$ & 419 & 370 \\
Amine number $(\mathrm{mg} \mathrm{KOH} / \mathrm{g})$ & 1.08 & 0 \\
PH value & 7.6 & 7.5 \\
\hline
\end{tabular}

\subsection{Internal Bond}

Six IB samples, three bending samples and two water-soak samples were tested from each fiberboard. The IB test results presented with average values with sample standard deviation information are shown in Table 4. There were variations among different samples from the same fiberboard. Since there were only three samples for MOE/MOR test, two samples for TS/WA tests, these data were presented with only average values. The densities of test samples were listed for reference.

IB strength reflects the quality of bonds between wood fibers and adhesives inside the fiberboard. Better bond quality usually results in higher IB value. As shown in Table 4 , IB of fiberboard bonded by pure pMDI resin was 0.89 $\mathrm{MPa}$, higher than that of most samples. When the $\mathrm{pMDI} /$ polyol mixture weight ratio was $75: 25$, the increase of recycled polyol in polyol mixture increased the IB value first and then decreased the IB value. At recycled polyol/commercial polyol weight ratio of 40: 60 , the IB value reached the highest value $(0.95 \mathrm{MPa})$. When using the recycled polyol as the solo polyol to bond wood, the IB value had the lowest value $(0.54 \mathrm{MPa})$. When the $\mathrm{pMDI} / \mathrm{polyol}$ mixture weight ratio was 50: 50, the trend of variation of IB values was similar. At recycled polyol/commercial polyol weight ratio of 40: 60, the IB value reached the highest value $(0.81 \mathrm{MPa})$. When using the recycled polyol as the solo polyol to bond wood, the IB value had the lowest value $(0.29$ $\mathrm{MPa})$. Generally, the IB values obtained at $\mathrm{pMDI} / \mathrm{polyol}$ mixture weight ratio of 75: 25 were higher than those obtained at pMDI/polyol mixture weight ratio of 50: 50 .

Table 4. Internal bond test results.

\begin{tabular}{llll}
\hline Recycled polyol/commercial polyol weight ratio & PMDI/polyol mixture weight ratio & Density $\left(\mathbf{g} / \mathbf{c m}^{\mathbf{3}}\right)$ & Internal bond $(\mathbf{M P a})$ \\
\hline- & $100: 0$ & 0.761 & $0.89(0.08)$ \\
$0: 100$ & $75: 25$ & 0.751 & $0.69(0.06)$ \\
$20: 80$ & $75: 25$ & 0.752 & $0.83(0.08)$ \\
$40: 60$ & $75: 25$ & 0.751 & $0.95(0.12)$ \\
$60: 40$ & $75: 25$ & 0.756 & $0.71(0.07)$ \\
$80: 20$ & $75: 25$ & 0.754 & $0.62(0.07)$ \\
$100: 0$ & $75: 25$ & 0.748 & $0.54(0.03)$ \\
$0: 100$ & $50: 50$ & 0.761 & $0.57(0.06)$ \\
$20: 80$ & $50: 50$ & 0.758 & $0.62(0.09)$ \\
$40: 60$ & $50: 50$ & 0.762 & $0.81(0.11)$ \\
$60: 40$ & $50: 50$ & 0.758 & $0.47(0.05)$ \\
$80: 20$ & $50: 50$ & 0.749 & $0.32(0.02)$ \\
$100: 0$ & $50: 50$ & 0.765 & $0.29(0.05)$ \\
\hline
\end{tabular}

Note: Values in parenthesis are sample standard deviations.

In fiberboard manufacturing, many factors might influence the physical and mechanical strength, such as adhesive type and application rate, hot press parameters, moisture content of mat, board dimension, press closing speed, etc. After pMDI was partially replaced by polyol mixture, under the condition of hot pressing, pMDI would react with polyol and fibers. The primary reaction might be-NCO groups with hydroxyl groups in wood and polyol, the minor reaction might be-NCO groups with water from wood and polyol [13]. These reactions both could result in the increase of the cross link density of cured adhesives which would contribute to the cohesion strength of cured pMDI resins [7]. In these cases, the polyol would help fiber bonding and increase IB strength. However, when the weight ratio of recycled polyol/ commercial polyo decreased from 75: 25 to 50: 50 , the percentage of recycled polyol in polyol mixture increased. In this case, pMDI became insufficient to react with hydroxyl groups and water to form bonds with high cross link density. Therefore, the IB values of fiberboards decreased.

\subsection{Modulus of Rupture and Modulus of Elasticity}

Bending property is to test the maximum compressive strength of fiberboards when they are bent to break. As shown in Table 3, MOR of fiberboard bonded by pure pMDI resin was $45 \mathrm{MPa}$, higher than that of most samples. When the pMDI/polyol mixture weight ratio was $75: 25$, the increase of recycled polyol in polyol mixture increased the MOR value first and then decreased the value. At recycled polyol/commercial polyol weight ratio of 40: 60 , the MOR value reached the highest value $(59 \mathrm{MPa})$. At recycled polyol/commercial polyol weight ratio of $60: 40$, the MOR value (46 MPa) was still comparable to that of fiberboard bonded by pure pMDI resin. When using the recycled polyol as the solo polyol to bond fiberboard, the MOR had the lowest value (39 MPa).

When the $\mathrm{pMDI} /$ polyol mixture weight ratio was 50: 50, the trend of variation of MOR values was similar. At recycled polyol/commercial polyol weight ratio of 40: 60 , the MOR value reached the highest value $(56 \mathrm{MPa})$. This value was higher than that of fiberboard bonded by pure pMDI, but lower than that of fiberboard bonded by adhesive when the recycled polyol/commercial polyol weight ratio was 60: 40 and the pMDI/polyol mixture weight ratio was 75: 25 . 
Generally, the MOR values obtained at pMDI/polyol mixture weight ratio of 75: 25 were higher than those obtained at pMDI/polyol mixture weight ratio of 50: 50 .

The MOE test results were similar to MOR results with maximum value obtained at recycled polyol/commercial polyol weight ratio of 40: 60. MOR and MOE results not only reflect the bonding performance, but also reflect surface condition of fiberboard, uniformity of adhesive application, and etc. Polyols could react with pMDI to improve bonding as discussed above. Meanwhile, the amine compounds in recycled polyols could also react with pMDI to form polyureas that could increase the cross link of adhesive and also increase the density of the surface of the fiberboard [14]. In this way, the addition of polyols, especially the recycled polyol, could help improve bending performance of fiberboard.

Table 5. Bending tests results.

\begin{tabular}{|c|c|c|c|c|}
\hline Recycled polyol/commercial polyol weight ratio & PMDI/polyol mixture weight ratio & Density $\left(\mathrm{g} / \mathrm{m}^{3}\right)$ & MOR (MPa) & MOE (MPa) \\
\hline- & 100: 0 & 0.755 & 45 & 6639 \\
\hline $0: 100$ & $75: 25$ & 0.751 & 41 & 6355 \\
\hline 20: 80 & $75: 25$ & 0.758 & 42 & 6410 \\
\hline 40: 60 & $75: 25$ & 0.759 & 59 & 6626 \\
\hline 60: 40 & $75: 25$ & 0.764 & 46 & 6450 \\
\hline 80: 20 & $75: 25$ & 0.758 & 41 & 5899 \\
\hline 100: 0 & $75: 25$ & 0.763 & 39 & 5317 \\
\hline 20: 80 & $50: 50$ & 0.749 & 42 & 5787 \\
\hline $40: 60$ & $50: 50$ & 0.758 & 56 & 6181 \\
\hline $60: 40$ & $50: 50$ & 0.751 & 40 & 6099 \\
\hline $80: 20$ & $50: 50$ & 0.750 & 36 & 5455 \\
\hline 100: 0 & $50: 50$ & 0.762 & 34 & 5289 \\
\hline
\end{tabular}

\subsection{Thickness Swell and Water Absorption}

TS value refers to the thickness incensement of fiberboard after immersed in the water of $25^{\circ} \mathrm{C}$ for $24 \mathrm{~h}$. WA value refers to the weight incensement of fiberboard after immersed in water of $25^{\circ} \mathrm{C}$ for $24 \mathrm{~h}$. TS and WA are usually used to evaluate the water resistance of fiberboards. Normally, the lower the TS and WA values are, the better the water resistance will be. As shown in table 6, TS and WA of fiberboard bonded by pure pMDI resin was $15.1 \%$ and $24.9 \%$, respectively. When the $\mathrm{pMDI} /$ polyol mixture weight ratio was 75: 25 , the increase of recycled polyol in polyol mixture decreased both the TS and WA values first and then increased these values. This result indicated that the increase of recycled polyol in polyol mixture improve water resistance of fiberboard first and then decreased the water resistance. At recycled polyol/commercial polyol weight ratio of 40: 60, both the TS and WA values reached the lowest $(14.1 \%$ and
$25.1 \%$, respectively). When using the recycled polyol as the solo polyol to bond fiberboard, both the TS and WA values had the highest value ( $16.2 \%$ and $28.1 \%$, respectively).

When the $\mathrm{pMDI} /$ polyol mixture weight ratio was 50: 50, the trends of TS and WA values were similar. Generally, both of the TS and WA values obtained at pMDI/polyol mixture weight ratio of 75: 25 were lower than those obtained at pMDI/polyol mixture weight ratio of 50: 50 .

Usually, TS and WA values are influenced by factors, such as condition of wood fibers, adhesive type and application rate, uniformity of adhesive application, fiberboard density, hot press parameters, etc [15]. Polyols contain many hydroxyl groups which are hydrophilic to water. Though most of the hydroxyl groups could be consumed by -NCO groups from pMDI resin, some hydroxyl groups would still remain and absorb water. The higher percentage the polyol used, the more the polyol would remain. In this case, the use of polyol did not obviously improve water resistance of fiberboard.

Table 6. Thickness swell and water absorption tests results.

\begin{tabular}{|c|c|c|c|c|}
\hline Recycled polyol/commercial polyol weight ratio & PMDI/polyol mixture weight ratio & Density $\left(\mathrm{g} / \mathrm{m}^{3}\right)$ & TS (\%) & WA $(\%)$ \\
\hline- & $100: 00: 00$ & 0.751 & 15.1 & 24.9 \\
\hline 0.069444444 & $75: 25: 00$ & 0.755 & 15.8 & 26.6 \\
\hline 0.888888889 & $75: 25: 00$ & 0.754 & 15.8 & 26.1 \\
\hline 40: 60 & $75: 25: 00$ & 0.756 & 14.1 & 25.1 \\
\hline 60:40:00 & $75: 25: 00$ & 0.753 & 14.5 & 26.5 \\
\hline $80: 20: 00$ & $75: 25: 00$ & 0.756 & 15.5 & 27.1 \\
\hline 0.069444444 & $50: 50: 00$ & 0.762 & 16.4 & 27.5 \\
\hline 0.888888889 & $50: 50: 00$ & 0.765 & 16 & 26.8 \\
\hline $40: 60$ & $50: 50: 00$ & 0.756 & 16.1 & 26.8 \\
\hline $60: 40: 00$ & $50: 50: 00$ & 0.758 & 17.5 & 30.3 \\
\hline $80: 20: 00$ & $50: 50: 00$ & 0.761 & 17.9 & 32.9 \\
\hline 100:00:00 & $50: 50: 00$ & 0.754 & 18.8 & 36.8 \\
\hline
\end{tabular}




\section{Conclusions}

In this study, PU foams from disposed insulation materials were collected and chemically decomposed to obtain the recycled polyol. The recycled polyol was mixed with commercial polyol at various weight ratios (0: 100, 20: 80, 40: 60, 60: 40, 80:20, and 100: 0) to prepare polyol mixtures. The obtained polyol mixtures were then sprayed with pMDI resin at various pMDI/polyol mixtures weight ratios (100: 0 , 75: 25 , and 50: 50) onto wood fibers to prepare fiberboards. The effects of recycled polyol/commercial polyol weight ratio and $\mathrm{pMDI} /$ polyol mixture weight ratio on physical and mechanical properties of bonded fiberboards were evaluated. The results showed that the addition of recycled polyol into commercial polyol improved the IB, MOR, and MOE strength of fiberboard, but their effects on water resistance of fiberboard were minor. When the recycled polyol/commercial polyol weight ratio was 40: 60 and the $\mathrm{pMDI} /$ polyol mixture was 25: 75, fiberboard had the overall best physical and mechanical performance.

In conclusion, the application of decomposed PU foams in fiberboard production provided a new possible way of recycling polymer wastes. The fiberboard bonded by pMDI resin and polyol mixtures had advantages of no formaldehyde emission, high mechanical properties, good water resistance, and low production cost.

\section{Author Contributions}

The Manuscript was written through contributions of all authors. All authors have given approval to the final version of the manuscript. Tongtong Cui and Yan Li contributed equally and should be considered as co-first authors.

\section{Conflicts of Interest}

The authors declare that they have no competing interests.

\section{Acknowledgements}

The authors are grateful for the supports of the Fundamental Research Funds for the Central Universities (Project No. BJFUKF201912), Youth Foundation of Shandong Natural Science Foundation (Project No. ZR2017QC006), Scientific Research Project of Colleges and Universities in Shandong Province (Project No. J17KA147) and National Student Innovation and Entrepreneurship Training Program (Project No. 201810434013).

\section{References}

[1] E. G. Ashton, W. Kindlein, R. Demori, L. H. A. Cândido, and R. Mauler, Recycling polymeric multi-material products through micronization. Journal of Cleaner Production, 116, 268-278, 2016.

[2] Y. Pang, X. Liu, T. Cui, Y. Li, Q. Li, H. Wan, X. Liu, and A.
Mao, Solvent decomposition of polyurethane foam obtained from waste upholstered furniture to recover polyols. American Journal of Polymer Science and Technology, 5 (3), 88-96, 2019.

[3] K. Hamad, M. Kaseem, and F. Deri, Recycling of waste from polymer materials: An overview of the recent works. Polymer Degradation and Stability, 98 (12), 2801-2812, 2013.

[4] C. C. Tamara, U. Lorena, J. T. Patrycja, S. Rafael, D. Janusz, and E. Arantxa, Thermoplastic polyurethane with glycolysate intermediates from polyurethane waste recycling. Polymer Degradation and Stability, 144, 411-419, 2017.

[5] D. Simon, A. M. Borreguero, A. Lucas, and J. F. Rodriguez, Recycling of polyurethanes from laboratory to industry, a journey towards the sustainability. Waste Management, 76, 147-171, 2018.

[6] Q. Li. M. Li, C. Chen, G. Cao, A. Mao, and H. Wan, Adhesives from polymeric methylene diphenyl diisocyanate resin and recycled polyols for plywood. Forest Products Journal, 67 (3/4), 275-282, 2017.

[7] A. Mao, R. Shmulky, Q. Li, and H. Wan, Recycling polyurethane materials A comparison of polyol from glycolysis with micronized polyurethane powder in particleboard applications. BioResources, 9 (3), 4253-4265, 2014.

[8] A. Mao, E. B. Hassan, and M. G. Kim, Investigation of low mole ratio UF and UMF resins aimed at lowering the formaldehyde emission potential of wood composite boards. BioResources, 8 (2), 2453-2469, 2013.

[9] A. Mao, E. B. Hassan, and M. G. Kim, Low mole ratio UF and UMF resins entailing uron-type methylene-ether groups and their low formaldehyde emission potentials. BioResources, 8 (2), 2470-2486, 2013.

[10] A. Mao, E. B. Hassan, and M. G. Kim, Low mole ratio urea-melamine-formaldehyde resins entailing increased methylene-ether group contents and their formaldehyde emission potentials of wood composite boards. BioResources, 8 (3), 4659-4675, 2013.

[11] A. Mao, E. B. Hassan, and M. G. Kim, The effects of adding melamine at different resin synthesis points of low mole ratio urea-melamine-formaldehyde (UMF) resins. BioResources, 8 (4), 5733-5748, 2013.

[12] Y. F. Pang, W. T. Xu, Q. Li, C. Li, and A. Mao, Research progress of bio-based wood adhesives. China Forest Products Industry, 45 (4), 3-7, 2018.

[13] X. Kong, G. Liu, and J. M. Curtis, Characterization of canola oil based polyurethane wood adhesives. International Journal of Adhesion and Adhesives, 31, 559-564, 2011.

[14] P. Berthevas, G. Santoro, R. Wevers, H. Gruenbauer, and A. Pizzi, Recycled polyurethane foam powder can be used in conjunction with pMDI in particle boards to obtain the required properties while reducing costs. 9th European Panel Products Symposium, Llandudno, Wales, UK, 40-47, 2005.

[15] P. Wei, X. Rao, J. Yang, Y. Guo, H. Chen, Y. Zhang, S. Chen, $\mathrm{X}$. Deng, and $\mathrm{X}$. Wang, Hot pressing of wood-based composites: a review. Forest Products Journal, 66 (7/8): 419-427, 2016. 